\title{
Reprodutibilidade intra e interobservadores da classificação de Fujiwara para degeneração do ligamento interespinhoso por meio de ressonância magnética e relação entre as lesões degenerativas do disco intervertebral e do ligamento interespinhoso
}

Intraobserver and interobserver reproducibility of Fujiwara rating of interspinous ligament by magnetic resonance imaging and relationship between lumbar disc degeneration and interspinous ligament degeneration

Reproducibilidad intra e interobservador de la clasificación de la degeneración de ligamento interespinoso Fujiwara por imágenes de resonancia magnética y la relación entre las lesiones degenerativas del disco intervertebral y del ligamento interespinoso

\section{Rafael de Carvalho Jorgetti' \\ Adriano Masayuki Yonezaki ${ }^{2}$ \\ Edgar Santiago Valesin Filho ${ }^{3}$ \\ Fabrício Ueno ${ }^{4}$ \\ Luciano Miller Reis Rodrigues ${ }^{5}$}

\section{RESUMO}

Objetivo: avaliar a aplicabilidade da classificação de Fujiwara em uma análise intra e interobservadores e compará-la com a classificação de Pfirrmann. Métodos: foram avaliados 87 ligamentos interespinhosos e discos intervertebrais de 29 pacientes (16 mulheres e 13 homens), com média de idade de 49 anos (24 a 78 anos), por meio da ressonância magnética pela classificação de Fujiwara e de Pfirrmann. A avaliação de Fujiwara foi realizada por três observadores, em duas ocasiões, com intervalo mí-

\begin{abstract}
Objective: to assess the clinical applicability of Fujiwara rating by intraobserver and interobserver analysis and to compare it to Pfirrmann rating of disc degeneration. Methods: eighty-seven interspinous ligaments and lumbar intervertebral discs of 29 patients (13 males and 16 females) with mean age of 49 years (24 to 81 years) were evaluated by magnetic resonance imaging by Fujiwara and Pfirrmann rating system. The assessment of Fujiwara rating was done by three observers in two
\end{abstract}

\section{RESUMEN}

Objetivo: evaluar la aplicabilidad de la clasificación en un análisis de Fujiwara intra-e interobservador $y$ compararla con la clasificación de Pfirrmann. Métodos: se evaluaron 87 ligamentos interespinosos y los discos intervertebrales de 29 pacientes (16 mujeres y 13 hombres), con edad media de 49 años (24 a 78 años), mediante resonancia magnética para la clasificación de Fujiwara y Pfirrmann. La evaluación de Fujiwara fue realizada por tres observadores, en dos ocasiones, con un intervalo de

\footnotetext{
Trabalho realizado na Disciplina do Aparelho Locomotor da Faculdade de Medicina do ABC - FMABC - Santo André (SP), Brasil.

'Médico Estagiário do Grupo de Coluna da Faculdade de Medicina do ABC - FMABC - Santo André (SP), Brasil. ${ }^{2}$ Médico Assistente do Grupo de Coluna da Faculdade de Medicina do ABC - FMABC - Santo André (SP), Brasil. ${ }^{3}$ Médico Colaborador do Grupo de Coluna da Faculdade de Medicina do ABC - FMABC - Santo André (SP), Brasil. ${ }^{4}$ Médico Assistente do Grupo de Coluna da Faculdade de Medicina do ABC - FMABC - Santo André (SP), Brasil. ${ }_{5}^{5}$ Mestre; Coordenador do Grupo de Coluna da Faculdade de Medicina do ABC - FMABC - Santo André (SP), Brasil.
} 
nimo de 15 dias. A avaliação de Pfirrman foi realizada pelos três observadores em um único tempo. Utilizamos o índice de Kappa para comparar a variação intra e interobservadores. Resultados: a concordância intraobservadores foi considerada boa; no entanto, a concordância interobservadores foi pobre, com índices de Kappa menores que 0,2. Conclusão: a classificação de Fujiwara não se mostrou reprodutiva para avaliação do ligamento interspinhoso. No entanto, a maior divulgação e utilização dessa classificação podem torná-la um bom instrumento de comparação entre resultados de diferentes estudos.

DESCRITORES: Classificação; Variações dependentes do observador; Imagem por ressonância magnética occasions, with a minimal interval of 15 days. The assessment of Pfirrmann rating was done by three observers at the same time. Statistical analyses were done by Kappa index to compare the intraobserver and interobserver variation. Results: the intraobserver agreement was considered to be good; however, the interobserver agreement was poor, with Kappa index lower than 0,2. Conclusion: the Fujiwara rating system showed no reproducibility to assess interspinous ligament. However, a larger disclosure and application of this rating system can turn this rating into a good instrument of comparison between an outcome of different studies.

KEYWORDS: Classification; Observer variation; Magnetic resonance imaging
15 dias. La evaluación de Pfirrman fue realizada por tres observadores en una sola vez. Se utilizó el índice Kappa para comparar intra e interobservador. Resultados: el acuerdo intraobservador fue bueno, pero el acuerdo interobservador fue pobre, con indices de Kappa inferiores al 0,2. Conclusión: la clasificación de Fujiwara no se mostró reproductiva para la evaluación del ligamento interespinoso. Sin embargo, la difusión más amplia y el uso de este tipo pueden hacer que sea una buena herramienta para comparar resultados de diferentes estudios.

DESCRIPTORES: Clasificación; Variaciones dependientes del observador; Imagen por resonancia magnética

\section{INTRODUÇÃO}

A prevalência de dor lombar na população geral é elevada, variando de 12 a $35 \%^{1}$ e acometendo igualmente homens e mulheres $^{2,3}$. Trata-se, portanto, de um importante problema de saúde pública, uma vez que consome enormes recursos tanto no seu diagnóstico como no tratamento. As manifestações clínicas da dor lombar resultam da interação de fatores anatômicos, psicológicos e sociológicos, que devem ser levados em conta no diagnóstico. Vale ressaltar que cerca de $10 \%$ dos pacientes acometidos tornam-se incapacitados ${ }^{4}$.

A dor lombar associa-se frequentemente à degeneração do disco intervertebral ${ }^{5}$, presente em cerca de $10 \%$ dos indivíduos na quinta década, e elevando-se para $60 \%$ dos indivíduos na sétima década ${ }^{6}$. Os processos de degeneração são complexos e envolvem deterioração das colunas anterior e posterior ${ }^{7}$. Kirkald-Willis et al. dividiram a cascata degenerativa em três fases: disfunção, instabilidade e reestabilização ${ }^{8}$. Esses autores identificaram e correlacionaram as alterações do disco com as das facetas articulares.

Além das lesões discais, a degeneração do ligamento espinhal posterior também tem sido associada à dor lombar',10, e vários estudos têm sido realizados visando esclarecer o papel dessas lesões na lombalgia.

Newman et al. realizaram um estudo anatômico abrangente sobre o ligamento interespinhoso, mostrando lesões degenerativas especialmente nos segmentos L4-L5 e L5$\mathrm{S} 1$ já na segunda década de vida ${ }^{11}$.
A presença de alterações anatômicas ou degenerativas nem sempre se traduz em sintomas clínicos. Porém, pode desencadear ou potencializar os sintomas de dor. Dessa forma, para um diagnóstico preciso, é fundamental empregar técnicas de imagem com boa resolução que permitam visualizar não só a morfologia da coluna, mas também as variações de cada doença, de traumas ou lesões degenerativas. A melhor técnica é a ressonância magnética, que analisa com precisão os tecidos moles, em especial os discos intervertebrais e os ligamentos espinhais ${ }^{12-17}$.

As classificações das lesões discais têm sido muito utilizadas, pois permitem comparação de casuísticas e orientam condutas clínicas. Dentre elas, a classificação de Pffirmann, que divide as lesões discais em cinco tipos, tem sido muito empregada devido à sua elevada reprodutibilidade ${ }^{18}$

Quanto às lesões ligamentares, são poucas as classificações disponíveis na literatura ${ }^{19}$. Fujiwara et al. elaboraram uma classificação na qual descrevem cinco tipos de lesões do ligamento interespinhoso ${ }^{10}$. O objetivo deste estudo foi aplicar a classificação proposta por Fujiwara em imagens obtidas de ressonância magnética (RM) de coluna de pacientes com dor lombar. No mesmo momento, realizamos também a classificação de Pffirman e comparamos os resultados das duas classificações. Analisamos ainda a variação inter e intraobservadores para a classificação de Fujiwara. 


\section{MÉTODOS}

Estudamos 29 pacientes com quadro de dor lombar associada ou não à ciatalgia, seguidos no ambulatório de Ortopedia do Hospital Estadual Mário Covas, Santo André (SP).

Após exame clínico, os pacientes realizaram exame de RM da coluna lombar. O aparelho utilizado era da marca Philips (Achieva Spine Coil), com resolução de 1-T. Foram obtidas imagens ponderadas em T1 e T2 sem supressão de gordura com os seguintes parâmetros: matriz, 336 x 512; espessura do corte de $4 \mathrm{~mm}$; espaço entre cortes, $1 \mathrm{~mm}$; número de excitações, 4; T2 FSE; TR 2478; TE 100; FOV 325/1,0. Para análise do ligamento interespinhoso utilizamos imagens obtidas no plano médio sagital.

Dois ortopedistas experientes (E1; E2) e um iniciante (I) analisaram em um único momento os discos intervertebrais, aplicando a classificação de Pfirmann (Quadro 1). Os mesmos ortopedistas analisaram os ligamentos interespinhosos aplicando a classificação de Fujiwara (Quadro 2) em duas ocasiões com intervalo de 15 dias.

\section{QUADRO 1 - Classificação de Pfirmann para avaliação do disco intervertebral}

\begin{tabular}{|c|c|c|c|c|}
\hline Grau & Estrutura & $\begin{array}{l}\text { Distinção } \\
\text { de ânulo } \\
\text { e núcleo }\end{array}$ & $\begin{array}{l}\text { Intensidade } \\
\text { de sinal }\end{array}$ & Altura do disco \\
\hline|| & $\begin{array}{l}\text { Homogênea, } \\
\text { brilho branco }\end{array}$ & Nítida & $\begin{array}{c}\text { Hiperintenso, } \\
\text { isointenso ao } \\
\text { liquor }\end{array}$ & Normal \\
\hline$\|$ & $\begin{array}{c}\text { Heterogêneo } \\
\text { com ou sem } \\
\text { bandas } \\
\text { horizontais }\end{array}$ & Nítida & $\begin{array}{l}\text { Hiperintenso, } \\
\text { isointenso ao } \\
\text { liquor }\end{array}$ & Normal \\
\hline |III & $\begin{array}{l}\text { Heterogêneo, } \\
\text { cinza }\end{array}$ & $\begin{array}{l}\text { Não } \\
\text { nítida }\end{array}$ & Intermediário & $\begin{array}{c}\text { Normal ou } \\
\text { pouco diminuída }\end{array}$ \\
\hline IV & $\begin{array}{l}\text { Heterogêneo, } \\
\text { cinza a preto }\end{array}$ & Perdida & $\begin{array}{l}\text { Intermediário } \\
\text { a hipointenso }\end{array}$ & $\begin{array}{l}\text { Normal a } \\
\text { moderadamente } \\
\text { diminuída }\end{array}$ \\
\hline V & $\begin{array}{l}\text { Heterogêneo, } \\
\text { preto }\end{array}$ & Perdida & Hipointenso & $\begin{array}{l}\text { Colapso do } \\
\text { espaço discal }\end{array}$ \\
\hline
\end{tabular}

\section{QUADRO 2 - Classificação de Fujiwara para} avaliação do ligamento interespinhoso

\begin{tabular}{|lc|}
\hline Grau & Características \\
\hline 1A & Baixa intensidade de sinal nas imagens em T1 e \\
& T2 sem hipertrofia do processo espinhoso \\
1B & Baixa intensidade de sinal nas imagens em T1 e \\
& T2 com hipertrofia do processo espinhoso \\
2 & Baixa intensidade de sinal nas imagens em T1 e \\
& alta intensidade de sinal em T2 \\
3 & Alta intensidade de sinal nas imagens em T1 e \\
4 & intermediário a alto sinal em T2 \\
4 & Outros sinais mistos \\
\hline
\end{tabular}

\section{Análise estatística}

Os resultados para idade estão expressos em média e desvio padrão. O índice Kappa foi utilizado para descrever a concordância intra e interobservadores.

Empregamos a padronização proposta por Landis e Kock $^{20}$ para o índice Kappa, ou seja, valores de Kappa compreendidos entre 0,8 e 1,0 significam que a concordância entre os observadores é excelente. Valores entre $0,6 \mathrm{e}$ 0,8 significam boa concordância; entre 0,4 e 0,6 , razoável concordância; entre 0,2 e 0,4 , pobre concordância; entre 0 e 0,2 , concordância sofrível; e entre - 1,0 e 0 , concordância extremamente pobre ou ausente.

\section{RESULTADOS}

Estudamos 29 pacientes (16 mulheres e 13 homens) com média de idade de 49,2 $\pm 5,9$ anos.

Desses pacientes, analisamos as imagens de três segmentos vertebrais, ou seja, 87 discos e ligamentos.

Na Tabela 1 estão descritos os resultados da análise intraobservadores.

TABELA 1 - Índice de Kappa intraobservadores aplicando a classificação de Fujiwara em três segmentos da coluna analisados em duas ocasiões com intervalo de 15 dias

\begin{tabular}{lccc}
\hline Observador & L3-L4 & L4-L5 & L5-S1 \\
\hline I & 0,2991 & 0,5229 & 0,5011 \\
E & 0,7862 & 0,6262 & 0,5625 \\
E2 & 0,5547 & 0,714 & 0,463 \\
\hline
\end{tabular}

A análise do segmento L4-L5 apresentou melhor concordância entre a primeira e a segunda análise entre os três observadores. No entanto, mesmo entre os ortopedistas mais experientes o índice Kappa não foi excelente, ou seja, a concordância entre as duas análises foi classificada como boa, enquanto para o ortopedista iniciante o índice foi classificado como razoável. Com exceção do observador 2 , o segmento que apresentou pior concordância entre as duas análises foi L3-L4, e o ortopedista iniciante apresentou o pior índice Kappa de todos os segmentos analisados. Em relação ao segmento L5-S1, a concordância entre as duas análises foi semelhante entre os três observadores; porém, foi classificada como razoável.

Na Tabela 2 estão resumidos os resultados da análise interobservador nos dois tempos do estudo. O ortopedista iniciante apresentou melhor concordância com o observador 1 do que com o observador 2, especialmente no segmento L4-L5, com melhora da concordância na segunda análise, o que também foi observado no segmento L5-S1. Vale a pena ressaltar que a concordância entre os dois ortopedistas mais experientes foi muito baixa nos dois tempos do estudo. 
TABELA 2 - Índice de Kappa interobservadores aplicando a classificação de Fujiwara em três segmentos da coluna analisados em duas ocasiões com intervalo de 15 dias

\begin{tabular}{lcccccc}
\hline & \multicolumn{2}{c}{ L3-L4 } & \multicolumn{2}{c}{ L4-L5 } & \multicolumn{2}{c}{ L5-S1 } \\
\hline & Tempo 1 & Tempo 2 & Tempo 1 & Tempo 2 & Tempo 1 & Tempo 2 \\
\hline I E 1 & 0,2648 & 0,0872 & 0,3044 & 0,4775 & 0,0996 & 0,2288 \\
I E2 & 0,076 & O,0876 & 0,055 & 0,1588 & 0,0865 & 0,2315 \\
E 1 $\times$ E2 & 0,0775 & N.A. & 0,0526 & 0,0754 & 0,0197 & 0,0363 \\
\hline
\end{tabular}

N.A.: Não avaliado

Quando comparamos os resultados da classificação de Pffirmann e Fujiwara realizados pelos três observadores, constatamos que o índice Kappa somente pode ser calculado para o ortopedista iniciante, ou seja, para o segmento L3-L4 esse índice foi de 0,0319 e para o segmento L5-S1, de 0,0295, revelando baixa concordância entre as duas classificações. Quanto aos resultados dos dois ortopedistas experientes, os resultados foram discrepantes a ponto de não ser possível calcular esse índice. Assim, a concordância entre as duas classificações não permite mais conclusões.

\section{DISCUSSÃO}

Apesar do aumento de estudos sobre degeneração do ligamento interespinhal, poucos analisam as características desse ligamento empregando $\mathrm{RM}^{10,21,22}$.

Em 2000, Fujiwara et al. propuseram uma classificação das lesões degenerativas do ligamento baseada na intensidade do sinal obtido de imagens da RM. Porém, sua reprodutibilidade não foi testada na ocasião ${ }^{10}$.

Recentemente, Keochorana et al. ${ }^{7}$ realizaram pequenas mudanças na classificação de Fujiwara ${ }^{10}$. Esses autores uniram os tipos $1 \mathrm{~A}$ e 4 de Fujiwara formando o tipo A. Já o tipo 2 de Fujiwara passou a ser o tipo C de Keochorana, e o tipo 3 tornou-se o tipo B. O grau D de Keochorana foi formado pelo tipo $1 \mathrm{~B}$, associado ou não a alterações de Modic para a placa terminal. Neste estudo, Keochorana et al. obtiveram uma excelente concordância intraobservador. Essa concordância, como esperado, diminuiu quando da análise interobservadores, mantendo-se, porém, dentro de valores satisfatórios.

No nosso estudo, utilizamos a classificação original de Fujiwara para graduar as lesões ligamentares. Obtivemos uma concordância intraobservador substancialmente menor do que a obtida por Keochorana, porém, considerada boa. A menor concordância entre um mesmo observador foi obtida pelo iniciante. Isso ocorreu provavelmente devido à menor experiência desse observador no estudo de imagens.

Quando realizamos a análise entre os observadores, diferentemente de Keochorana, não obtivemos concordância significativa. No entanto, o bom nível de concordância intraobservador e a concordância satisfatória entre o observador I e o observador E1 no segmento L4-L5 mostram que a classificação de Fujiwara pode ser útil na avaliação das lesões degenerativas dos ligamentos interespinhosos. Então, a baixa concordância entre os três observadores e entre o observador E2 e os outros observadores separadamente se deve à pouca experiência do grupo na aplicação da classificação de Fujiwara, que ficará mais apurada quando a mesma for utilizada como rotina. A classificação das lesões ligamentares associada com as queixas clínicas dos pacientes também deve contribuir para validar a classificação de Fujiwara.

A análise dos nossos resultados revelou que houve melhor concordância nos níveis comumente mais afetados pelo processo degenerativo, ou seja, a maior concordância ocorreu no segmento L4-L5 seguido pelo segmento L5S1. Isso sugere que a classificação de Fujiwara pode ser mais reprodutível quando se analisam ligamentos mais degenerados.

Ao comparar as classificações de Pfirrmann e Fujiwara, não foi possível estabelecer qualquer correlação entre a degeneração do disco intervertebral e do ligamento interespinhoso.

\section{CONCLUSÃO}

Nossos resultados demonstraram que a classificação de Fujiwara não se mostrou reprodutiva na avaliação do ligamento interespinhoso. No entanto, a maior divulgação e uso dessa classificação podem torná-la um bom instrumento de comparação de resultados de diferentes estudos.

\section{REFERÊNCIAS}

1. Maniadakis N, Gray A. The economic burden of back pain in the UK. Pain. 2000;84(1):95-103.
2. Svensson HO, Andersson GB. The relationship of low-back pain, work history, work environment, and stress: a retrospective cross-sectional study of 38- to 64 year-old women. Spine (Phila Pa 1976). 1989;14(5):517-22.
3. Raj PP. Intervertebral disc: anatomyphysiology-pathophysiologytreatment. Pain Pract. 2008;8(1):18-44.

4. Svensson HO, Andersson GB. Low back pain in 40- to 47-year-old men: work history and work environment factors. Spine (Phila Pa 1976). 1983;8(3):272-6. 
5. Luoma K, Riihimäki H, Luukkonen R, Raininko R, Viikari-Juntara E, Lamminen A. Low back pain in relation to lumbar disc degeneration. Spine (Phila Pa 1976). 2000;25(4):487-92.

6. Miller JA, Schmatz C, Schultz AB. Lumbar disc degeneration: correlation with age, sex, and spine level in 600 autopsy specimens. Spine (Phila Pa 1976). 1988;13(2):173-8.

7. Keorochana G, Taghavi C E, Tzeng ST, Lee KB, Liao JC, Yoo JH, et al. MRI classification of interspinous ligament degeneration of the lumbar spine: intraobserver and interobserver reliability and the frequency of disagreement. Eur Spine J. 2010;19(10):1740-5.

8. Kirkaldy-Willis WH, Wedge JH, Yong-Hing K, Reilly J. Pathology and pathogenesis of lumbar spondylosis and stenosis. Spine (Phila Pa 1976). 1978;3(4):319-28.

9. Jinkins JR. Acquired degenerative changes of the intervertebral segments at and suprajacent to the lumbosacral junction. A radioanatomic analysis of the nondiscal structures of the spinal column and perispinal soft tissues. Eur J Radiol. 2004;50(2):134-58.

10.Fujiwara A, Tamai K, An HS, Shimizu K, Yoshida H, Saotome K. The interspinous ligament of the lumbar spine. Magnetic resonance images and their clinical significance. Spine (Phila Pa 1976). 2000;25(3):358-63.

11. Newman PH. Sprung Back. J Bone Joint Surg Br.1952;34(1):30-7.
12. Grenier N, Greselle JF, Vital JM, Kien P, Baulny D, Broussin J, et al. Normal and disrupted lumbar longitudinal ligaments: correlative MR and anatomic study. Radiology 1989;171(1):197-205.

13. Grenier N, Kressel HY, Schiebler ML, Grossman RI, Dalinka MK. Normal and degenerative posterior spinal structures: MR imaging. Radiology. 1987;165(2):517-25.

14.Petersilge CA, Pathria MN, Emery SE, Masaryk TJ. Thoracolumbar burst fractures: evaluation with MR imaging. Radiology. 1995;194(1):4954.

15.Silverman CS, Lenchik L, Shimkin PM, Lipow KL. The value of MR in differentiating subligamentous from supraligamentous lumbar disk herniations. AJNR Am J Neuroradiol. 1995;16(3):571-9.

16.Terk MR, Hume-Neal M, Fraipont M, Ahmadi J, Colletti PM. Injury of the posterior ligament complex in patients with acute spinal trauma: evaluation by MR imaging. AJR Am J Roentgenol. 1997;168(6):1481-6.

17. Tracy PT, Wright RM, Hanigan WC. Magnetic resonance imaging of spinal injury. Spine (Phila Pa 1976). 1989;14(3):292-301.

18.Ueno FH, Rodrigues, LMR, Filho ESV, Puertas EB. Intraobserver and interobserver reproductibility of Pfirrmann rating of disc degeneration by magnetic resonance imaging. Coluna/Columna. 2008;7(4):330-3.
19.Goobar JE, Sartoris DJ, Hajek PC, Baker LL, Haghighi P, Hesselink J, et al. Magnetic resonance imaging of the lumbar spinous process and adjacent soft tissues: normal and pathologic appearances. J Rheumatol.1987;14(4):788-97.

20.Landis JR, Koch GG. The measurement of observer agreement for categorical data. Biometrics. 1977;33(1):159-74.

21.Maes R, Morrison WB, Parker L, Schweitzer ME, Carrino JA. Lumbar interspinous bursitis (Baastrup disease) in a symptomatic population: prevalence on magnetic resonance imaging. Spine (Phila Pa 1976). 2008;33(7):E211-5.

22.Scapinelli R, Stecco C, Pozzuoli A, Porzionato A, Macchi V, De Caro R. The lumbar interspinous ligaments in humans: anatomical study and review of the literature. Cells Tissues Organs. 2006;183(1):1-11.

\section{Correspondência}

Rafael de Carvalho Jorgetti

Avenida Cidade Jardim, 3990

Bosque dos Eucalíptos

CEP: $12232-001$ - São Paulo (SP), Brasil

E-mail: gonzera@yahoo.com.br 